\title{
Optical Measurements of Shock Waves in Critical Nozzles at Low Reynolds Numbers
}

\author{
Soichiro Yagi, Saya Inoue, Shinichiro Nakao, Daisuke Ono, Yoshiaki Miyazato \\ Faculty of Environmental Engineering, The University of Kitakyushu, Kitakyushu, Japan \\ Email: capra15pcx@gmail.com
}

How to cite this paper: Yagi, S., Inoue, S., Nakao, S., Ono, D. and Miyazato, Y. (2017) Optical Measurements of Shock Waves in Critical Nozzles at Low Reynolds Numbers. Journal of Flow Control, Measurement \& Visualization, 5, 36-50.

https://doi.org/10.4236/jfcmv.2017.52003

Received: March 3, 2017

Accepted: April 14, 2017

Published: April 17, 2017

Copyright $\odot 2017$ by authors and Scientific Research Publishing Inc. This work is licensed under the Creative Commons Attribution International License (CC BY 4.0).

http://creativecommons.org/licenses/by/4.0/

\begin{abstract}
Two-dimensional critical nozzle flows at low Reynolds numbers are visualized by the rainbow schlieren deflectometry. Experiments have been performed in a region of overexpanded nozzle flow. The variation of the shock structure against the back pressure ratio can be clearly visible with color gradation. Static pressure rises due to the shock-induced flow separation are compared with the previous theories. The unsteady characteristics of overexpanded critical nozzle flows at low Reynolds numbers are quantitatively and qualitatively visualized using laser schlieren and Mach-Zehnder interferometer systems combined with a high-speed digital camera. It was found that an oscillating normal shock wave appears inside the nozzle, and that the shock wave has a specified dominant frequency. Also the time-history of the oscillating shock wave is obtained from both the systems and compared with each other.
\end{abstract}

\section{Keywords}

Compressible Flow, Micro Nozzle Flows, Optical Flow Visualization, Rainbow Schlieren Deflectometry, Mach-Zehnder Interferometry, Shock Wave Oscillation, Flow Separation

\section{Introduction}

A critical nozzle provides a reliable and precise way of measuring mass flow rate and it has an important role in many industrial applications including mass flow controllers, calibration standards for other gas flow meters, and pressure isolators. When the ratio of back pressure downstream of a critical nozzle to the upstream stagnation pressure is maintained below a critical threshold known as the critical back pressure ratio, the mass flow rate through the nozzleremains constant and it is entirely independent of any changes in flow conditions downstream of the nozzle throat. In the ISO Standard 9300, the design and usage of 
critical nozzles are well described over a range of throat Reynolds numbers from $10^{5}$ to $10^{7}$ and the divergent section just downstream of the nozzle throat plays a key role functioning as a pressure recovery element that extends the flow range capabilities of the device by increasing the minimum critical back pressure ratio required to reach a choked flow condition. However, in critical nozzles with significantly smaller throat diameter, relatively large fluctuations in the mass flow through the nozzles have been observed, making the calibration of these nozzles difficult. In the past, although flow properties through supersonic nozzles have been extensively studied in the research areas of rocket propulsion, the geometrical size, and therefore the Reynolds numbers of these nozzles were significantly higher than considered in the present study.

Von Lavante et al. [1] [2] numerically and experimentally studied the flow behavior in a representative critical Venturi nozzle as recommended by the ISO Standard 9300 over a range of theoretical Reynolds numbers from $1.0 \times 10^{5}$ to $1.0 \times 10^{7}$. At the throat Reynolds numbers of $1.0 \times 10^{5}$ and $5.0 \times 10^{5}$ with a back pressure ratio for a normal shock wave to stay in the nozzle evaluated under the isentropic flow conditions except the shock, they numerically showed that pressure disturbances can propagate upstream beyond the nozzle throat, even above the critical back pressure ratio, thus leading to fluctuating mass flow rate through the nozzle. Kim et al. [3] numerically investigated the effects of back pressure fluctuations on the critical Venturi nozzle flows and showed that for low Reynolds numbers the pressure disturbances from the nozzle exit can propagate upstream beyond the nozzle throat, giving rise to the significant fluctuations in mass flow rate through the nozzle, while, for high Reynolds number, the pressure signals occurring at the nozzle exit do not propagate upstream of the throat. Nakao and Takamoto [4] experimentally investigated choking phenomena of ISO-type critical Venturi nozzles for the Reynolds number range from 40 to 30,000 lower than the ISO Standard 9300. They showed that the minimum Reynolds number satisfying the choking condition is about 40 and the critical back pressure ratio is only about 0.05 at this minimum Reynolds number and the critical back pressure ratio has a local maximum value around $R e_{\mathrm{th}}=4000$ and that the local minimum value is around $R e_{\text {th }}=10,000$ due to the change in characteristics of the boundary layer in the divergent section of the nozzle. Also, they demonstrated that the critical back pressure ratio is a function of the theoretical Reynolds number only, not dependent on the throat diameter. Furthermore, the change of the discharge coefficients occurs abruptly when the back pressure ratio approaches the critical value, and the distribution of the discharge coefficient against the back pressure ratio has at least one local maximum and one local minimum when the back pressure ratio is above the critical value and they explained the phenomena in terms of the interaction between the shock wave and the boundary layer in the diffuser, as Von Lavante et al. [1] [2] showed in their numerical simulation.

Lower back pressure ratios to fulfill a purely supersonic flow in the divergent section downstream of a nozzle throat will stabilize the mass flow rate through 
the nozzle. Ideally, when a nozzle is operated with overexpansion, the flow in the nozzle is supersonic over the entire region downstream of the throat, and the static pressure at the exit plane is uniformly lower than the back pressure. However, a detailed and deep comprehension of critical nozzle flows operating under low Reynolds numbers with overexpansion remains to be fully elucidated and little is known in current literature because of technical difficulties in measurements. The real nozzle flows with overexpansion at higher Reynolds numbers often involve the flow separation in a section between the throat and the nozzle exit as a result of the interaction of a shock wave with a boundary layer, because the boundary layer cannot very frequently endure the abrupt pressure gradient due to the shock wave. Under such conditions the flows expand inside the nozzle to a location just upstream of the shock wave and then compressed to a specified back pressure and the theory to predict the pressure rise due to the shock-induced boundary layer separation proposed by Arens et al. [5] has been widely used in the design of supersonic nozzles operated at overexpanded conditions.

The objective of the present study is to investigate experimentally flow behavior in a critical Venturi nozzle by optical flow visualization and the interaction of a shock wave with a boundary layer at low Reynolds numbers are investigated by a comparison of the present experiments by the rainbow schlieren deflectometry with the past theories including that of Arens et al. [5]. Also a MachZehnder interferometer system [6] combined with a high speed digital camera is, for the first time, applied to a two-dimensional unsteady flow field including shock waves in the overexpanded nozzle flows at low Reynolds numbers. The measurement of an oscillating shock wave in the nozzle by a high speed laser schlieren system has been performed to validate that by the Mach-Zehnder interferometer.

\section{Experimental Setup and Method}

\subsection{Rainbow Schlieren Technics}

The experiments were carried out in a blowdown facility with a rainbow schlieren optical system, and performed for a range of nozzle back pressure ratios from 0.55 to 0.65 , which are included in a range of overexpanded nozzle flows. The Reynolds number employed in the present paper was varied from $7.5 \times 10^{4}$ to $8.9 \times 10^{4}$ and it is defined based on the assumption of the ideal flow through a nozzle under choked flow conditions as $R e_{\mathrm{th}}=\rho^{*} u^{*} H_{\mathrm{th}} / \mu_{\mathrm{os}}$ where $\rho^{*}$ and $u^{*}$ are the density and velocity at the nozzle throat, respectively, $H_{\mathrm{th}}$ is the throat height, and $\mu_{\mathrm{os}}$ is the coefficient of viscosity evaluated under stagnation conditions at nozzle inlet. It is referred to as the throat Reynolds number in the research areas on critical nozzle flows. The Reynolds numbers in the present study are lower than the range of the Reynolds number covered by ISO 9300. As illustrated in Figure 1, the present nozzle has the same wall contour as the toroidal throat Venturi nozzle recommended by ISO 9300 and a constant width of $12 \mathrm{~mm}$ over the whole length from the inlet to the exit. The rainbow schlieren system consists of rail-mounted optical components including a $50 \mu \mathrm{m}$ diameter pinhole, 


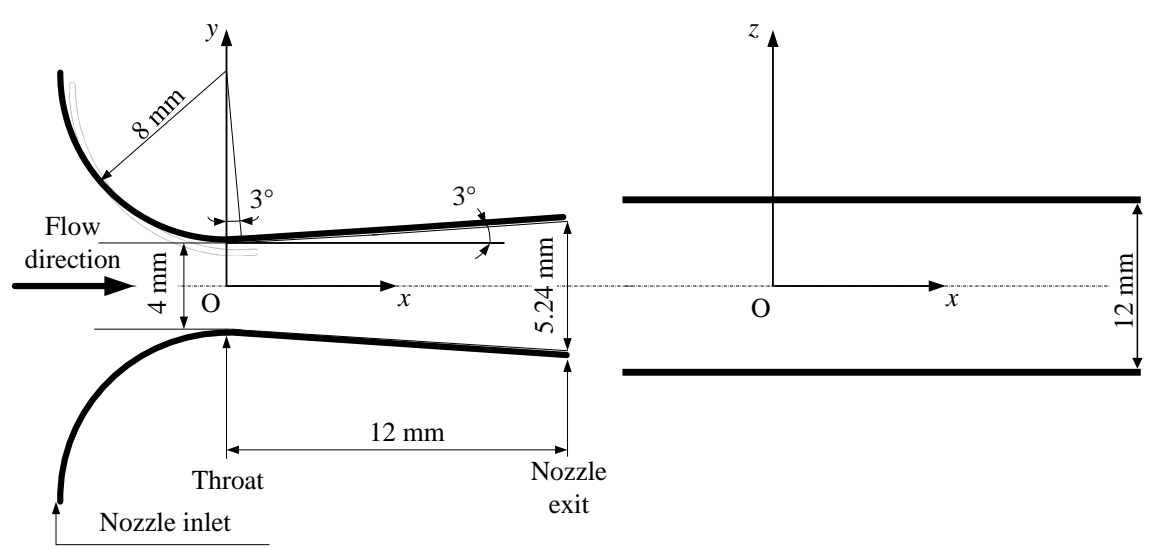

(a)

(b)

Figure 1. Schematic drawing of nozzle used for experiment. (a) Front view, (b) Plan view.

two $100 \mathrm{~mm}$ diameter, $500 \mathrm{~mm}$ focal length achromatic lenses, a computer generated $35 \mathrm{~mm}$ wide slide with color gradation in a $1.4 \mathrm{~mm}$ wide strip, and a digital camera with variable focal length lens. A continuous $250 \mathrm{~W}$ metal halide light source connected to a $50 \mu \mathrm{m}$ diameter fiber optic cable provides the light input at the pinhole through a $16.56 \mathrm{~mm}$ focal length objective lens. The camera output in the RGB format is digitalized by a personal computer with 24 bit color frame grabber. A detailed description for a calibration method of the rainbow filter is contained in the paper by Takano et al. [7].

\subsection{Mach-Zehnder Interferometer Systems}

The experiments have been carried out in a blow down facility with a laser schlieren optical system and Mach-Zehnder interferometers, and performed for a range of nozzle operating conditions from $p_{\mathrm{b}} / p_{\mathrm{os}}=0.45$ to 0.65 which are included in a region of overexpanded nozzle flow where $p_{\mathrm{os}}$ is the plenum pressure and $p_{\mathrm{b}}$ is the back pressure. The Reynolds number of the present study was varied from $7.5 \times 10^{4}$ to $1.1 \times 10^{5}$. These conditions are lower than the range of the Reynolds number covered by ISO 9300. As illustrated in Figure 1, the wall contour of the present nozzle is the same shape as the toroidal throat Venturi nozzle recommended by ISO 9300.

As shown in Figure 2, a He-Ne laser is used as a light source for laser schlieren and Mach-Zehnder interferometer systems and these systems are combined with a high-speed digital camera with $30 \mathrm{k}$ frames per second. Visualization of the critical nozzle flows for each operating pressure ratio was performed and recorded in 2000 pictures. The effective spatial resolution of the present imaging system is $25 \mathrm{pixel} / \mathrm{mm}$ or $0.04 \mathrm{~mm} / \mathrm{pixel}$. Using these systems for two-dimensional nozzle flows including a shock wave, the instantaneous shock position, the frequency and amplitude of the shock wave, and the two-dimensional unsteady flow field can be measured with ease. For each laser schlieren image, the shock wave is defined to be positioned at the steepest negative grey scale gradient. Since a shade level of video output is essentially dependent on the intensity of schlieren light source and cut off of the light at knife edge, the shock image with 


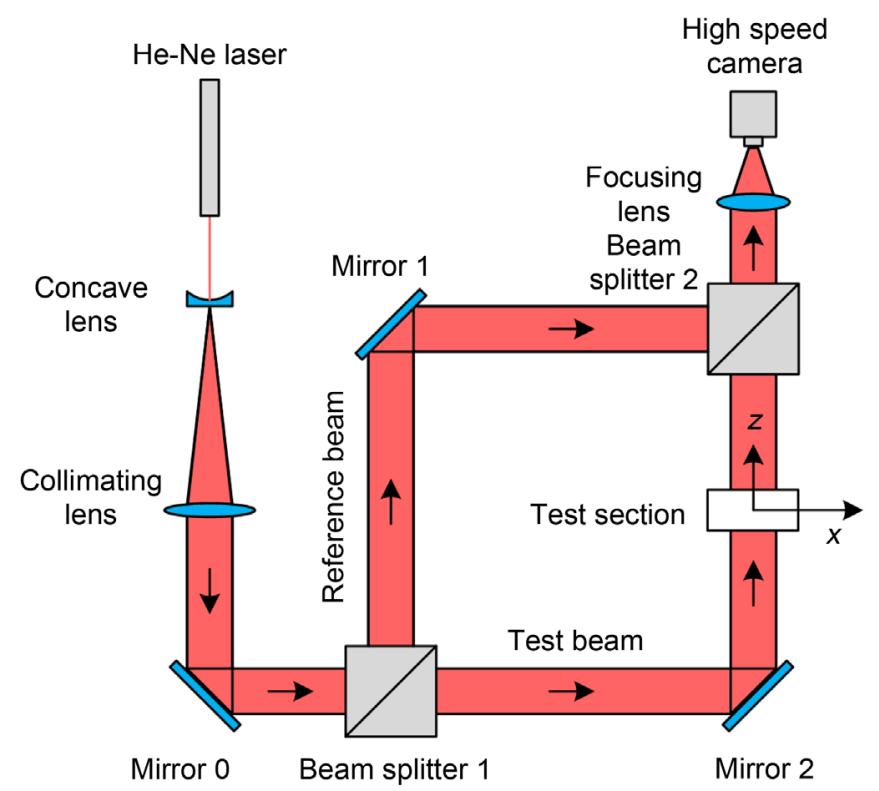

Figure 2. Experimental setup for Mach-Zehnder Interferometer system.

comparatively constant intensity were obtained at the height of the nozzle centerline in all the present test conditions where the shapes of the shock wave were maintained nearly normal to the streamwise direction.

On the other hand, Mach-Zehnder interferometers can estimate the unsteady two-dimensional characteristics of the whole flow field in the nozzle including the shock displacement by analyzing fringe shift from the reference image. Since the wedge fringe method can measure density more minutely than the infinite fringe method by analyzing a fringe shift, in the present work the wedge fringe method is employed. The analytical procedure of an interferogram is divided into two steps, which are the fringe shift analysis and density calculation from the fringe shift. The former is done using the Fourier transform method, which is presented by Takeda et al. [8] and can obtain a fringe shift two-dimensionally with a high space resolution. The latter is done under the assumption that a flow field is two-dimensional. The quantitative methodology of extracting the twodimensional density field in the test section is described below.

In the wedge fringe method, the mirrors and beam splitters are deliberately misaligned to produce a background fringe pattern of straight lines. In the present experiment, the background fringes were made perpendicular to the nozzle axis by appropriate adjustment of the beam splitter 2 shown in Figure 2. When a flow with a refractive-index disturbance is introduced in the test section, the background fringes are changed into deformed fringe patterns. On a screen or recording media for a fixed time, the typical profiles showing background and deformed fringe patterns are illustrated in Figure 3 as lines of constant phase. The parallel and equally spaced fringes shown as red solid lines in Figure 3 are also referred to as wedge fringes. This interval $b$ (see Figure 3) between two successive crests of the background fringes is a function of the intersection angle 

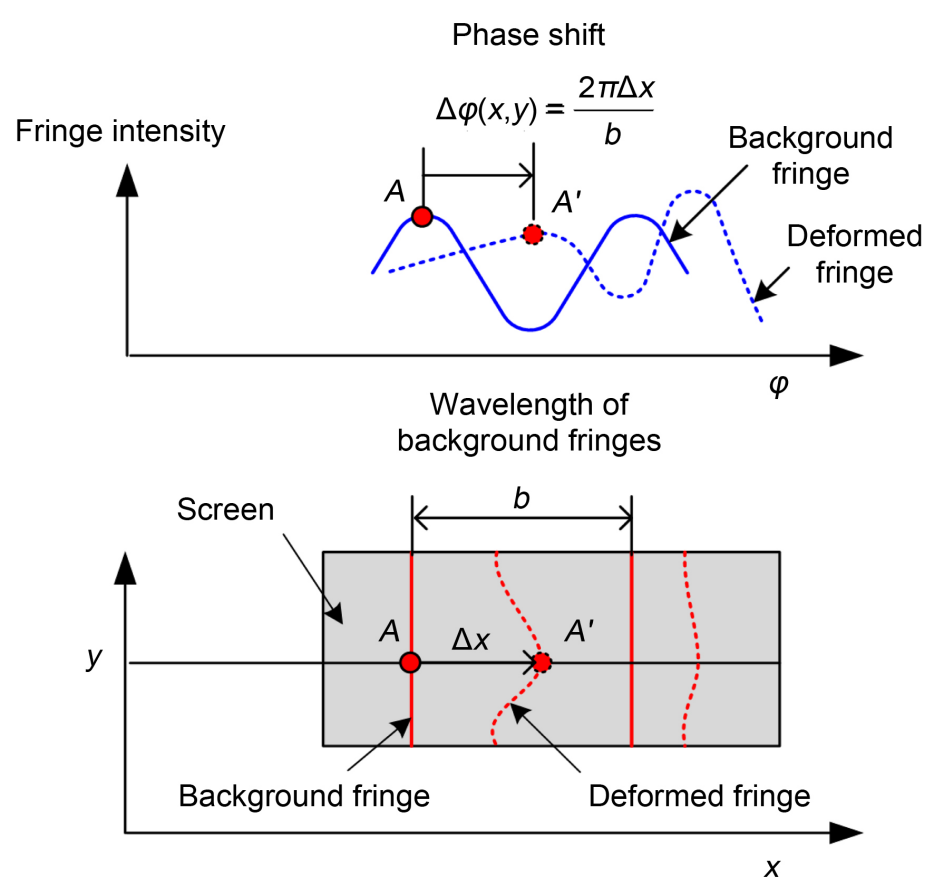

Figure 3. Cross section of nozzle.

between reference and test beams and the wavelength of the laser light used. The intensity profile $g(x, y)$ of the deformed fringe pattern can be given by

$$
g(x, y)=g_{0}(x, y)+g_{1}(x, y) \cos \left[k_{0} x+\Delta \varphi(x, y)\right]
$$

with the phase shift $\Delta \varphi(x, y)$ containing the desired information on the density field in the test section where $g_{0}(x, y)$ and $g_{1}(x, y)$ represent unwanted irradiance variations arising from the nonuniform light reflection or transmission when the test beam is passing through the test section, and $k_{0}=2 \pi / b$. The coordinates $x$ and $y$ form the vertical plane, which is perpendicular to the test beam propagation direction, and the $z$-axis is taken as the direction in which the test beam propagates.

Equation (1) is rewritten in the following expression

$$
g(x, y)=g_{0}(x, y)+c(x, y) \exp \left(i k_{0} x\right)+c^{*}(x, y) \exp \left(-i k_{0} x\right)
$$

with

$$
c(x, y)=\frac{1}{2} g_{1}(x, y) \exp [\operatorname{i} \Delta \varphi(x, y)]
$$

where $i$ is the imaginary number and the asterisk ${ }^{\star}$ denotes a complex conjugate.

The Fourier transform of Equation (2) with respect to $x$ is given by

$$
G(k, y)=G_{0}(k, y)+C\left(k-k_{0}, y\right)+C^{*}\left(k+k_{0}, y\right)
$$

where the capital letters denote the Fourier transforms of the primitive functions, respectively and $k$ is the spatial wave number in the $x$ direction. Since the spatial variations of $g_{0}(x, y), g_{1}(x, y)$, and $\Delta \varphi(x, y)$ are slow compared with the spatial frequency $k_{0}$ when the interval between fringes is sufficiently small, the Fourier spectra in Equation (4) are separated by the wave number $k_{0}$ and have 
the three independent peaks, as schematically shown in Figure 4(a). We make use of either of the two spectra on the carrier, say $C\left(k-k_{0}, y\right)$, and translate it by $k_{0}$ on the wave number axis toward the origin to obtain $C(k, y)$, as shown in Figure 4(b). The unwanted background variation $G_{0}(k, y)$ has been filtered out in this stage by a pertinent band pass filter. We compute the inverse Fourier transform of $C(k, y)$ with respect to the $k$ to obtain $c(x, y)$, defined by Equation (3). Then we calculate a complex logarithm of Equation (3) as follows:

$$
\ln c(x, y)=\ln \frac{g_{1}(x, y)}{2}+i \Delta \varphi(x, y)
$$

From Equation (5), we can obtain the phase shift $\Delta \varphi(x, y)$ in the imaginary part completely separated from the unwanted amplitude variation $g_{1}(x, y)$ in the real part. The phase shift $\Delta \varphi(x, y)$ is related to the density field through the Gradstone-Dale formula as follows:

$$
\frac{\Delta \varphi(x, y)}{2 \pi}=\frac{K}{\lambda_{0}} \int_{-L / 2}^{L / 2}\left[\rho(x, y)-\rho_{a}\right] \mathrm{d} z
$$

where $L=12 \mathrm{~mm}$ is the spanwise length of the test section, $\rho_{a}$ is the atmospheric density, $\lambda_{0}$ is the wavelength of a light source in vacuum, and $K=2.2587 \times 10^{-4}$ $\mathrm{m}^{3} / \mathrm{kg}$ is the Gradstone-Dale constant. Considering the two-dimensional refractive index field, i.e., independent of the $z$ axis, the density $\rho(x, y)$ in the flow field is expressed by

$$
\rho(x, y)=\rho_{a}+\frac{\lambda_{0}}{2 \pi K L} \Delta \varphi(x, y)
$$

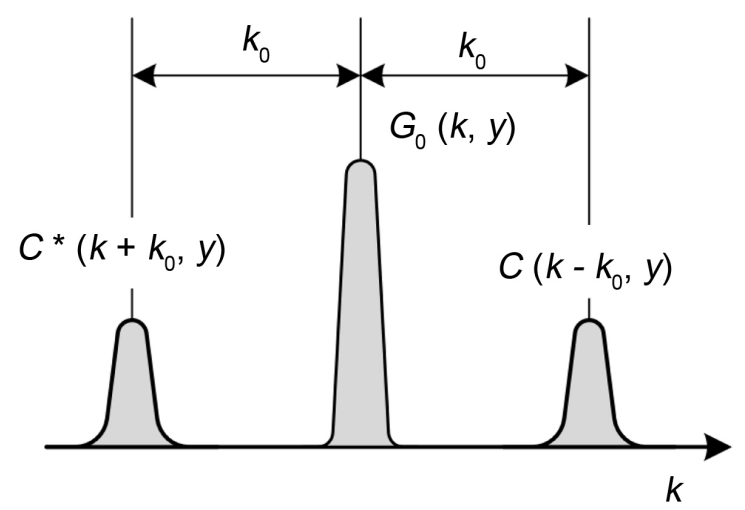

(a)

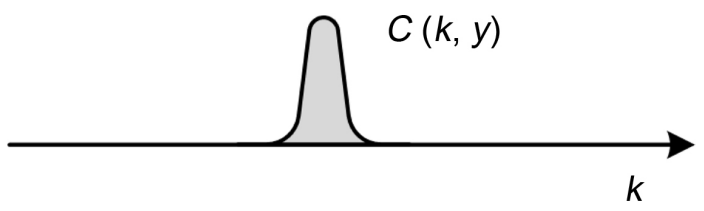

(b)

Figure 4. Fourier-transform method of fringe-pattern analysis. (a) Separated fourier spectra; (b) Parallel translation of single spectrum selected. 
Since Equation (7) shows that the phase shift at a fixed time is directly proportional to the flow density at the same time, characteristics of an unsteady flow field including a shock wave can be evaluated by examining the time history of the fringe shift.

An image of the flow field in the test section by the Mach-Zehnder interferometer is formed onto the CMOS sensor of a high-speed digital camera (Photron, FASTCAM SA1.1) which records a JPEG RGB image (24-bit each color) at a resolution of $640 \times 272$ square pixels. The plane of focus is located in the middle of the test section. The RGB image is then turned into an 8-bit grayscale image by a linear transformation. Therefore, the distributions of background and deformed fringes with 256 different possible intensities can be calculated from the Mach-Zehnder images for the density-field in the test section.

\section{Results and Discussion}

\subsection{Rainbow Schlieren Experiment}

Rainbow schlieren pictures of overexpanded nozzle flow are shown in Figure 5 with the flow from left to right where the rainbow filter is vertically set to view the shape of the shock waves. The ratio $p_{\mathrm{b}} / p_{\mathrm{os}}$ of back pressure $p_{\mathrm{b}}\left(p_{\mathrm{b}}\right.$ is the same value as the atmospheric pressure) to plenum pressure $p_{\text {os }}$ was decreased in steps of 0.05 from $p_{\mathrm{b}} / p_{\mathrm{os}}=0.65$ to 0.50 . Generally, no shock wave exists inside the nozzle for the overexpanded flow condition based upon the assumption of one-dimensional isentropic theory except for a normal shock wave. However, as can be seen in Figure 5(a), a nearly normal shock wave stands at a location just downstream of the nozzle throat. Proceeding from top to bottom, the shock moves toward the nozzle exit and the shock structure progressively varies from nearly normal shock to X-type shock train which implies that the boundary layer flow separation occurs at the foot of the shock.

To verify the shock-induced flow separation, the experimental data are

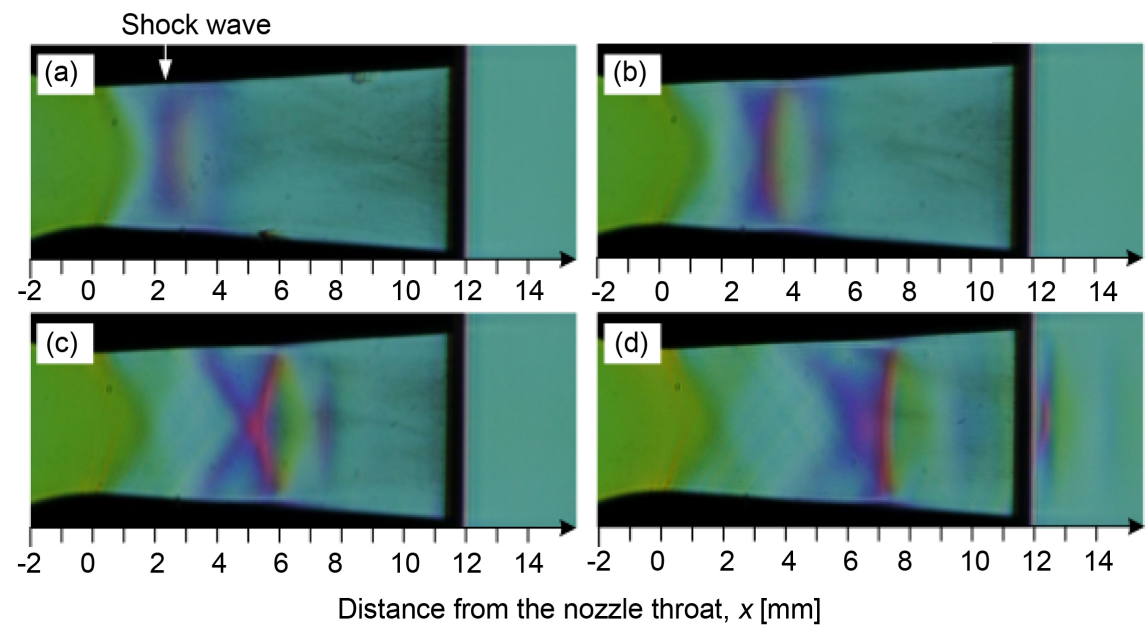

Figure 5. Schlieren pictures: (a) $p_{\mathrm{b}} / p_{\mathrm{os}}=0.65$, (b) $p_{\mathrm{b}} / p_{\mathrm{os}}=0.60$, (c) $p_{\mathrm{b}} / p_{\mathrm{os}}=0.55$, (d) $p_{\mathrm{b}} / p_{\text {os }}=0.50$. 
compared with the past theories. Figure 6 shows the variation of the shock location $x_{1}$ along the nozzle centerline ( $x$ axis) from the nozzle throat against the back pressure ratio $p_{\mathrm{b}} / p_{\text {os }}$ where the $x_{1}$ for a shock train was measured as the distance parallel to the $x$ axis from the throat to the foot of the leading shock of the first shock. The density gradient across a shock wave increases in the flow direction when the flow passes through the shock wave. Since a large density gradient leads to the large transverse displacement of the light ray at the rainbow filter plane, the position of the leading shock from the nozzle throat was estimated from the streamwise hue variations using the calibration curve for the rainbow filter used in the experiment.

The dash-dotted line in Figure 6 indicates the theoretical solution derived from the Rankine-Hugoniot relation for a normal shock location in the present nozzle where the back pressure ratios when a normal shock just stands at the nozzle exit and at the throat become 0.654 and 0.837 , respectively. The dashed and solid lines show the exact solutions on the static pressure ratio across an oblique shock based upon the assumption that the flow separation occurs at the flow deflection angle equal to the nozzle wall half-angle and the static pressure $p_{\mathrm{s}}$ just behind the shock is equal to the back pressure $p_{\mathrm{b}}$. They correspond to weak and strong shock solutions, respectively. In addition the dotted line in Figure 6 shows the theoretical curve proposed by Arens and Spiegler [5] where the static pressure $p_{\mathrm{s}}$ after the shock-induced flow separation is assumed to be the same as the back pressure $p_{\mathrm{b}}$. The symbols A to D correspond to the schlieren pictures of Figures 5(a)-(d), respectively. Figure 6 shows that the experimental values for a nearly single normal shock shown at Points A and B are in good agreement with the strong shock solution. The theoretical solutions shown in Figure 6 don't

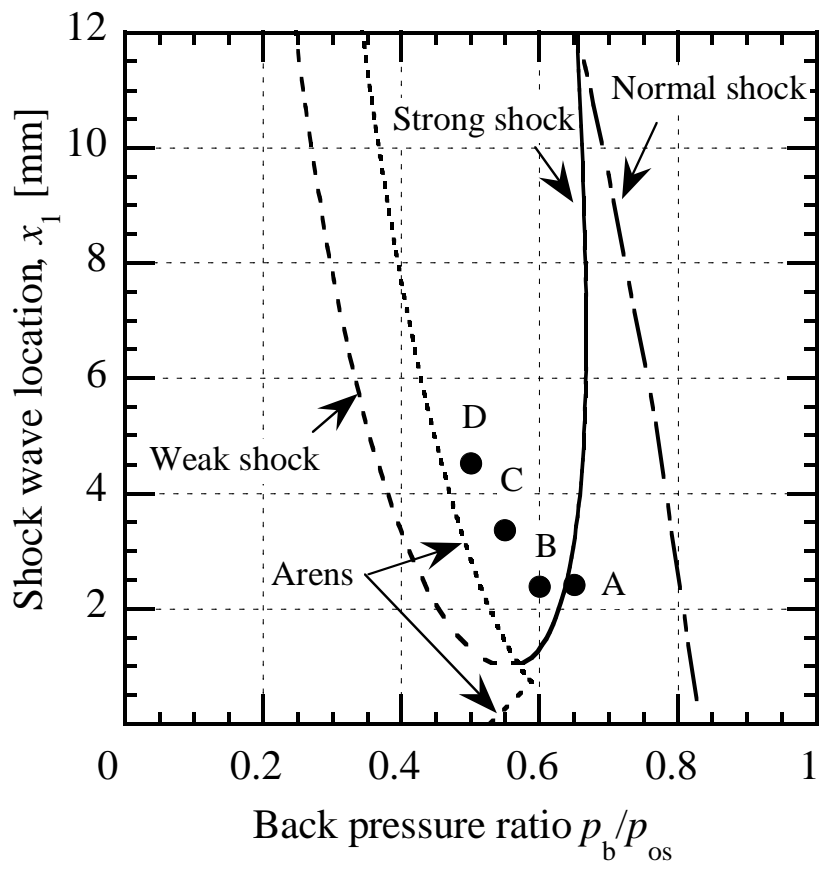

Figure 6. Variation of shock location. 
provide any exact prediction about the experimental values for shock trains at Points $\mathrm{C}$ and $\mathrm{D}$. The reason why these differences appear seems to come from the boundary layer condition. Arens and Spiegler [5] attempt to predict the turbulent shock wave-boundary layer separation. They found that the best agreement with the experimental data for the shock pressure ratio required to separate a turbulent boundary layer is obtained by using the assumption that the pressure rise must be sufficient to stagnate a characteristic velocity in the boundary layer. This theoretical curve agrees well with the experimental data caused by the turbulent shock wave-boundary layer separation. However, the throat Reynolds numbers in the present study is quite smaller than those of the previous research. Therefore, the differences between the theoretical curve proposed by Arens and Spiegler and the experimental plots obtained from the present study may come from the estimation of the velocity profile in the boundary layer.

The separation pressure ratio $p_{1} / p_{\mathrm{s}}$ is plotted against the separation Mach number $M_{1 \mathrm{e}}$ in Figure 7 where $M_{1 \mathrm{e}}$ is calculated on the basis of isentropic expansion from plenum chamber to the shock location $x_{1}$, and $p_{1}$ is the static pressure at the $x_{1}$, and $p_{\mathrm{s}}$ is the static pressure after the compression due to the shock-induced flow separation. Although the separation pressure $p_{\mathrm{s}}$ cannot be obtained in the present experiment, the $p_{\mathrm{s}}$ is assumed to be the same as the back pressure based on the assumption of Arens et al. [5]. The validation is performed by a comparison between the present experimental results and the theory of Arens et al. The theory of Arens et al. shown in Figure 6 and Figure 7 has extensively been used in research areas of rocket propulsion to predict the exact location of the shock-induced boundary layer separation in a supersonic nozzle with a diffuser divergent angle held almost constant in the downstream direction. Their theory assumes that the $p_{\mathrm{s}}$ is the same as the back pressure downstream of the nozzle, i.e., no pressure recovery occurs after the separation.

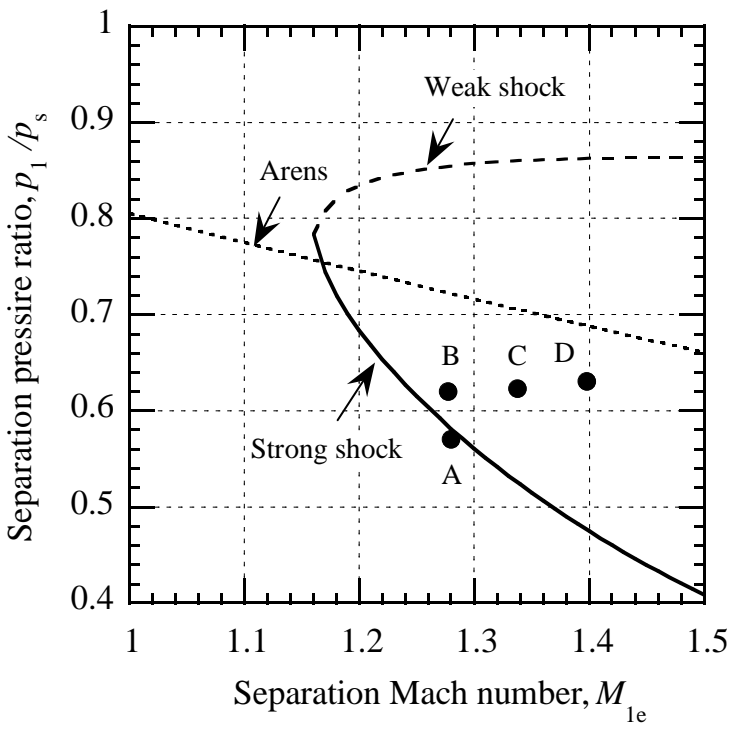

Figure 7. Separation pressure ratio. 
In our experiments, the $p_{\mathrm{s}}$ itself cannot be directly obtained, but the ratio $p_{\mathrm{s}} / p_{1}$ or $p_{1} / p_{\mathrm{s}}$ can be obtained when the static pressure $p_{1}$ just upstream of the shock or the corresponding freestream Mach number at the shock location is known.

Figure 7 shows that the flow separations at Points $\mathrm{A}$ and $\mathrm{B}$ are deemed to be responsible at the adverse pressure gradient by the shock wave only, not the interaction between the shock wave and boundary layer. The flow separation in the case depends only on the free stream Mach number just upstream of the shock wave because the shock strength is a function of the Mach number only. On the other hand, the flow separations at Points C and D are caused by the interaction between the shock wave and the boundary layer because a shock train appears as a consequence of the interaction between a normal shock wave and a boundary layer [9]. The separation pressure ratios for shock trains remain a constant value regardless of the separation Mach numbers. According to a lot of experimental research in the past, a shock train is produced in a confined duct for the free stream Mach numbers beyond around 1.5 [9]. However, the present study shows a shock train appears for the free stream Mach numbers below 1.5 and it may be considered as an effect of the lower Reynolds number, as compared to that of the previous research, in other words, a thinner velocity profile in the boundary layer at the location of the shock. This leaves room for further investigation.

\subsection{Laser Schlieren and Mach-Zehnder Interferometer Experiments}

Only the result for a nozzle operating condition of 0.65 is discussed in detail in the present paper. Typical laser schlieren image of an instantaneous flowfield in a critical nozzle at $p_{\mathrm{b}} / p_{\text {os }}=0.65$ is shown in Figure 8 where spatial resolution of the shock position is $0.04 \mathrm{~mm}$ in the streamwise direction. Generally, no shock wave exists inside the nozzle for overexpanded flow conditions. However, as shown in Figure 8, a nearly normal shock wave stands at the location just downstream of the throat. A normal shock wave was observed in much of the 2000 successively-recorded schlieren images.

Figure 9 displays the time history of a shock wave location in case of $p_{\mathrm{b}} / p_{\mathrm{os}}=$ 0.65 . The light intensity from the laser schlieren is directly proportional to the density gradient in the flow. In the present laser schlieren, an increase in density

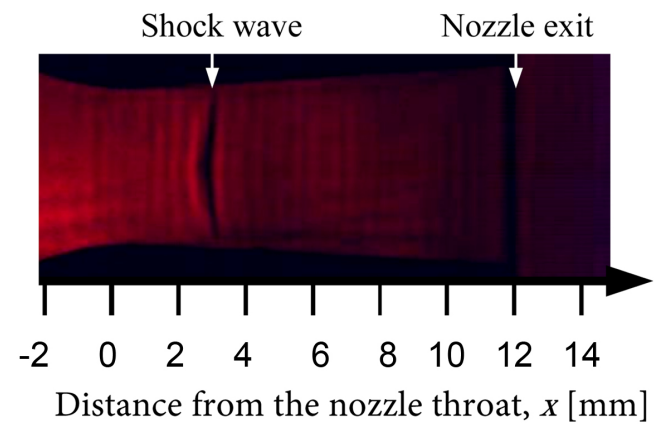

Figure 8. Schlieren image for $p_{\mathrm{b}} / p_{\mathrm{os}}=0.65$. 


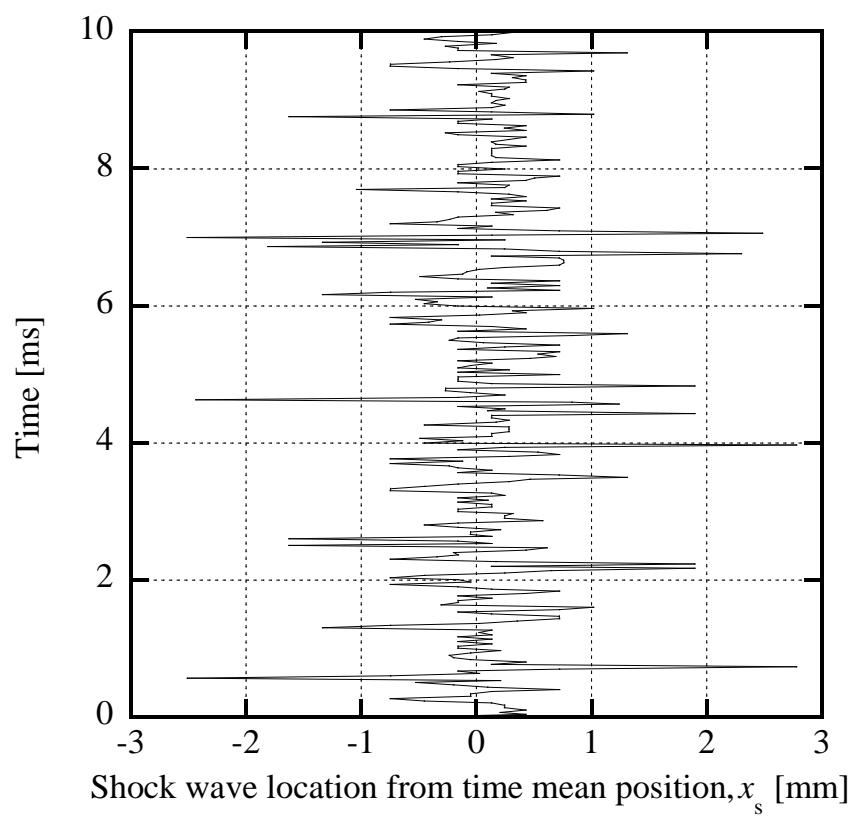

Figure 9. Time history of shock wave location.

gradient can be seen as a drop in light intensity and vice versa. In other words, whenever a shock wave is present in the flow field, a large drop followed by an increase in light intensity is observed. The time history of an oscillating shock can be obtained from such light intensity variation in the flow field. The $x_{s}=0$ shows the time-mean location of an oscillating shock in the nozzle where the origin of the time averaged shock wave location is identical with a location of $x=$ $3.17 \mathrm{~mm}$. The shock wave vibrates across a time-mean position. Figure 10 shows the power spectrum density distribution (PSD) of the shock displacement calculated using the experimental data of Figure 9. The area under the curve of the PSD is identical to the variance of the shock displacement along the nozzle centerline.

The PSD shows that the oscillating shock has a maximum value of $3 \mathrm{kHz}$ with a narrowband spectrum. Figure 11(a) and Figure 11(b) show typical interferogram of nozzle flow in the case of $p_{\mathrm{b}} / p_{\mathrm{os}}=1.0$ (background image) and $p_{\mathrm{b}} / p_{\mathrm{os}}=$ 0.65 , respectively. A uniform brightness image can be seen in Figure 11(a). In Figure 11(b), the fringes near the shock wave and upper and bottom walls are distorted notably. It is known that fringe shift depends only on density in the wedge fringe method. Therefore, the time history of the two-dimensional density field can be calculated by analyzing the fringe shift obtained from Figure 11. Figure 12 shows the power spectrum density distribution (PSD) of the fringe shift fluctuation at the time-mean shock location in Figure 11. As can be seen in Figure 12, the dominant frequency is $3 \mathrm{kHz}$ and it is in good quantitative agreement with that obtained from the laser schlieren. Von Lavante et al. [1] [2] numerically solved the unsteady behavior of a critical Venturi nozzle flow at a Reynolds number of $1.0 \times 10^{5}$ and showed that pressure fluctuations at the downstream of the nozzle exit propagate upstream beyond the position of the 


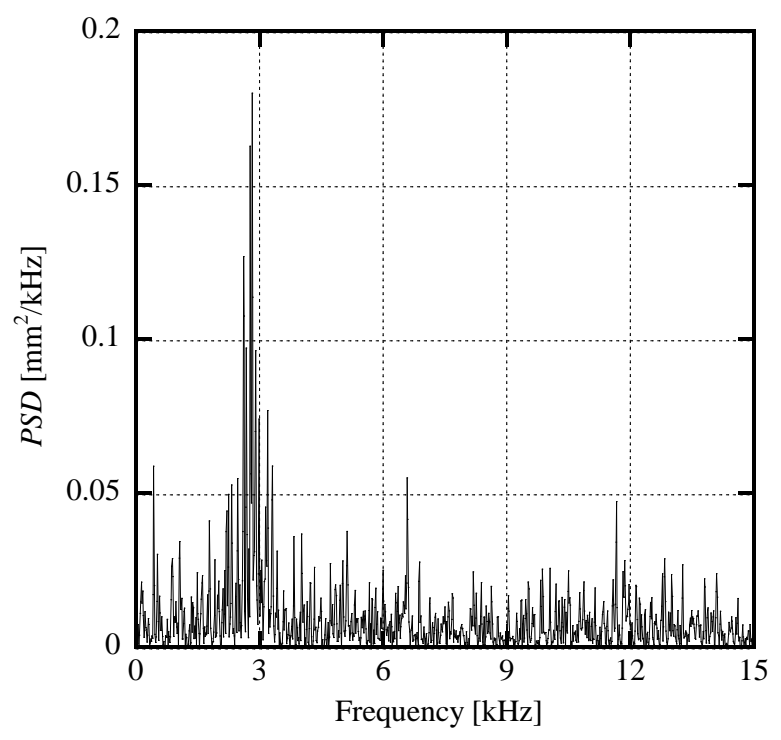

Figure 10. PSD of shock oscillation.

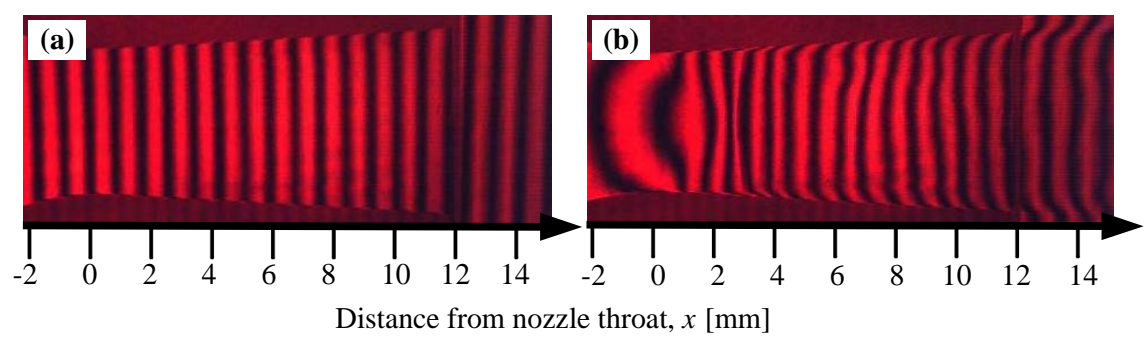

Figure 11. Typical interferogram: (a) $p_{\mathrm{b}} / p_{\mathrm{os}}=1.0$ (background image), (b) $p_{\mathrm{b}} / p_{\mathrm{os}}=0.65$.

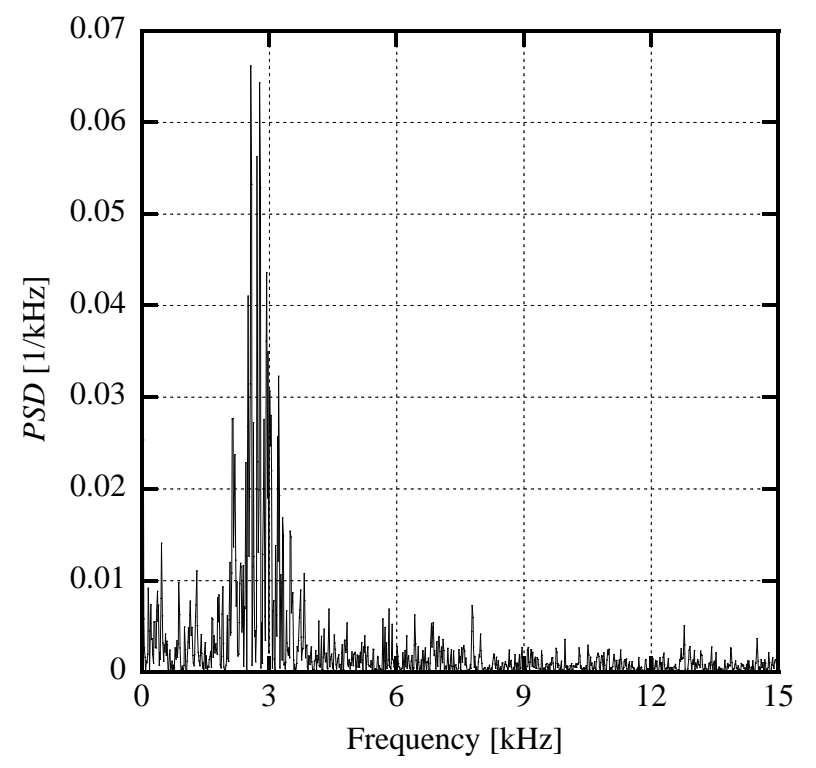

Figure 12. PSD of fringe shift fluctuation at mean shock location $(x=3.17 \mathrm{~mm})$.

nozzle throat. As a result, an intermittent decrease of the mass flow rate occurs because of periodical unchoking at the nozzle flow. As can be seen in Figure 10 
and Figure 12, the present study shows a shock wave oscillating across a mean position with a dominant frequency of $3 \mathrm{kHz}$.

\section{Concluding Remarks}

Overexpanded nozzle flows at low Reynolds numbers were visualized by a rainbow schlieren optical system. Schlieren pictures show that the shock gradually moves toward the nozzle exit from the location just downstream of the nozzle throat with decreasing back pressure ratio and its structure progressively changes from nearly normal shock to shock train. Also, the separation pressure ratio for a single normal shock is in good agreement with the theoretical solution for a strong oblique shock, while that for shock trains remains a constant value. Critical nozzle flows at low Reynolds numbers have been experimentally investigated in a region of overexpanded nozzle flow by using laser schlieren and MachZehnder interferometer systems combined with a high-speed digital camera. As a result, a nearly normal shock wave stands at the location just downstream of the nozzle throat and it oscillates at a dominant frequency of around $3 \mathrm{kHz}$ at a Reynolds number of $7.5 \times 10^{4}$. Furthermore, the unsteady two-dimensional characteristics of the critical nozzle flows can be obtained by the present MachZehnder interferometer system. As a result, two characteristics of critical nozzle flows at low Reynolds numbers were obtained from the present work. Firstly, a nearly normal shock wave appears in the overexpanded nozzle flow and it oscillates with a dominant frequency of around $3 \mathrm{kHz}$. Secondly, a shock train appears for free stream Mach numbers below 1.5 and it may be considered as an effect of the lower Reynolds number, as compared to that of the previous research [9]. This result may come from a thinner velocity profile in the boundary layer at the location of the shock. These phenomena would be explained by the interaction between the shock wave and the boundary layer. However, the detailed research would be required to clarify the mechanism of the periodic oscillation of the shock wave at low Reynolds numbers. The present results will provide reliable experimental data to validate numerical simulations of critical nozzle flows at low Reynolds numbers. In addition, this study demonstrates the capability of the two-dimensional measurements in an unsteady flow field by the Mach-Zehnder interferometer.

\section{Acknowledgements}

The authors would like to gratefully acknowledge the assistance of graduate students Shota GOTO, Kazuki KITAMURA, and Atsushi MATSUYAMA of the University of Kitakyushu for their invaluable support to the experimental work and exceptional skills in the fabrication of the facility.

\section{References}

[1] Von Lavante, E., Zachcial, A., Nath, B. and Dietrich, H. (2000) Numerical and Experimental Investigation of Unsteady Effects in Critical Venturi Nozzles. Flow Measurement and Instrumentation, 11, 257-264. 
[2] Von Lavante, E., Zachcial, A., Nath, B. and Dietrich, H. (2001) Unsteady Effects in Critical Nozzles Used for Flow Metering. Measurement, 29, 1-10.

[3] Kim, H., Kim, J., Park, K., Setoguchi, T. and Matsuo, S. (2004) Study of the Effects of Unsteady Downstream Conditions on the Gas Flow through a Critical nozzles. Proceedings of the Institution of Mechanical Engineers, Part C: Journal of Mechanical Engineering Science, 218, 1163-1173. https://doi.org/10.1243/0954406042369053

[4] Nakao, S. and Takamoto, M. (2000) Choking Phenomena of Sonic Nozzles at Low Reynolds Numbers. Flow Measurement and Instrumentation, 11, 285-291.

[5] Arens, M. and Spiegler, E. (1963) Shock-Induced Boundary Layer Separation in Over-Expanded Conical Exhaust Nozzles. AIAA Journal, 1, 578-581. https://doi.org/10.2514/3.1598

[6] Simith, A. and Lim, T. (2000) Flow Visualization. Imperial College Press, Covent Garden, London, 226-231.

[7] Takano, H., Kamikihara, D., Ono, D., Nakao, S., Yamamoto, H. and Miyazato, Y. (2016) Three-Dimensional Rainbow Schlieren Measurements in Underexpanded Sonic Jets from Axisymmetric Convergent Nozzles. Journal of Thermal Science, 25, 78-83. https://doi.org/10.1007/s11630-016-0836-0

[8] Takeda, M., Ina, H. and Kobayashi, S. (1982) Fourier-Transform Method of FringePattern Analysis for Computer-Based Topography and Interferometry. Journal of the Optical Society of America, 72, 156-160. https://doi.org/10.1364/JOSA.72.000156

[9] Matsuo, K., Miyazato, Y. and Kim, H. (1999) Shock Train and Pseudo-Shock Phenomena in Internal Gas Flows. Progress in Aerospace Sciences, 35, 33-100.

Submit or recommend next manuscript to SCIRP and we will provide best service for you:

Accepting pre-submission inquiries through Email, Facebook, LinkedIn, Twitter, etc. A wide selection of journals (inclusive of 9 subjects, more than 200 journals)

Providing 24-hour high-quality service

User-friendly online submission system

Fair and swift peer-review system

Efficient typesetting and proofreading procedure

Display of the result of downloads and visits, as well as the number of cited articles

Maximum dissemination of your research work

Submit your manuscript at: http://papersubmission.scirp.org/

Or contact jfcmv@scirp.org 